\section{GP206 MALARIA - EXPERIENCE OF A PAEDIATRICS DEPARTMENT IN AN UNDERDEVELOPED COUNTRY}

${ }^{1}$ Marta Pinheiro*, ${ }^{1}$ Vanessa Gorito, ${ }^{2}$ Rosa Carneiro, ${ }^{2}$ Paula Gonçalves, ${ }^{2}$ Natércia Duarte. ${ }^{1}$ Department of Paediatrics. Centro Materno Pediátrico, Centro Hospitalar e Universitário de São João, Oporto, Portugal; '2Department of Paediatrics. Hospital Geral José Macamo, Maputo, Mozambique

\subsection{6/archdischild-2019-epa.265}

Introduction Malaria is a parasitic disease (Plasmodium) transmitted by Anopheles mosquitoes. The diagnose is made after a positive result in the rapid diagnostic test or the presence of the parasite in the blood smear. Complicated malaria, classified with clinical and laboratory criteria, is a medical emergency and presupposes injectable treatment. In endemic countries it is a serious problem of public health and resource consumption. The objective of this study was intended to the reality of Paediatrics of a hospital in Mozambique.

Material and Methods Retrospective and descriptive review of hospitalizations in paediatric age (0-14 years) for malaria in a general hospital of an underdeveloped and endemic country from July/2017 to June/2018.

Results Five hundred and fifty-one children were admitted $22 \%$ of hospitalizations in the pediatric ward, 54\% were male, with 3 years of median ages. They presented with complications $53.4 \%$ of patients - mainly cytopenias and convulsive episodes - and $17.4 \%$ with other concomitant infections. Initially, artesunate intravenous was administered to all patients, they were discharged, on average 3 days later, with oral therapy; registered 10 cases of drug resistance. One patient was transferred to the central hospital (cerebral malaria). There were no deaths.

Discussion Data obtained are concordant with published epidemiology, reinforcing the impact of this pathology in the health services. Paediatric age is one of the risk groups, which always requires hospitalization under 5 years. Non-endemic countries should take advantage of recently updated strategies of the World Health Organization and Public Health departments of high-prevalence countries to do properly in imported cases.

\section{GP207 A PAIN IN THE NECK; A COMPLETED AUDIT OF USE OF THROAT CULTURES IN A PAEDIATRIC EMERGENCY DEPARTMENT}

Jennifer Geraghty*, Fionnuala Caulfield, Maeve Mc Cormack. Department of Paediatrics, Our Lady of Lourdes Hospital, Drogheda, Ireland

\subsection{6/archdischild-2019-epa.266}

Background The National Institute for Clinical Excellence (NICE) published an innovation briefing on 'point-of-care diagnostic testing for suspected strep A infection in sore throat' in May 2018. This suggested that use of these tests in additional to clinical scoring systems could assist in rapid decision making, reduce unnecessary antibiotic use and provide additional patient reassurance compared with throat culture.

Aims To audit use of throat culture and rate of positive results in the paediatric emergency department of a secondary paediatric hospital. To raise awareness of the importance of using clinical scoring systems in assessing the need for throat culture. To identify whether the introduction of point of care testing for Group A streptococcal infection (GAS) would be feasible in this setting.
Methods Emergency department records were used to retrospectively identify patients with acute sore throat who had a throat culture taken over a three month period. This was followed by interventions in the form of education sessions and visual reminders placed in the emergency department on the modified centor criteria clinical scoring system for GAS. We then re-audited throat cultures taken over a one month period.

Results In the initial three month audit period 53 throat cultures were taken. Of these one $(1.8 \%)$ was positive for GAS. Following the interventions 31 throat cultures were taken in the one month period of which four (12.9\%) were positive for GAS.

Conclusion Throat cultures were used in the assessment of children with acute sore throat despite their limited assistance in clinical decision making in the emergency department setting. There was a very low yield of positive throat culture results in these patients. Raised awareness of clinical scoring systems did increase the yield of positive throat culture results. Further interventions are needed to reduce the rate of negative throat cultures before the benefit of introducing rapid antigen tests can be assessed.

\section{GP208 THE PUBLIC HEALTH RESPONSE TO A BLOOD EXPOSURE INCIDENT IN A LOCAL SECONDARY SCHOOL}

${ }^{1}$ Abigail Collins, ${ }^{2}$ Una Fallon, ${ }^{2}$ Fiona McGuire, ${ }^{2}$ Margaret Cosgrove, ${ }^{2}$ Michelle Connolly, ${ }^{3}$ Heather Burns* ${ }^{1}$ Health Services Executive, Dublin, Ireland; ${ }^{2}$ Health Services Executive, Tullamore, Ireland; ${ }^{3}$ Health Services Executive, Department of Public Health, Midlands, Tullamore, Ireland

10.1136/archdischild-2019-epa.267

The Public Health Department (PHD) was contacted by a local secondary school of an incident regarding a shared needle exposure the previous week. A group of students had undertaken a project to investigate the effects of exercise on blood glucose levels, as part of a national school science competition. The students undertook the experiments during three consecutive lunch breaks. A student with diabetes carried out the testing and used the same lancing devices on all children, with no hygiene or infection control considerations. On the final day a teacher became aware of the incidents. After several days, Public Health were contacted by the school for advice.

Public Health urgently visited the school in question to discern further details. After an initial risk assessment, an incident management team (IMT) was convened within the PHD to best manage the acute response to the incident, and to take actions as required. Our incident entailed all children being both potentially recipient and donor of a blood borne infection. No national or international guidelines consulted offered advice for this scenario. The clinical team returned to the school the following day to meet the parents of the children involved, to assess further the child's risk of being a donor of a bloodborne virus, and to explain that their child might be both a source and a recipient of a blood borne virus. Nationally produced information sheets were tailored by the IMT for this setting, to give to all students and parents. All children consented to have their Hepatitis B, C and HIV serology tested and were offered early vaccination against Hepatitis B by the IMT in school. The outcome for all children was uneventful. 
The IMT identified deficits in the school's knowledge and management of biohazards, and had concerns this could occur in another school. The IMT created a poster to inform schools and child care facilities about biohazards and their appropriate management. The IMT contacted the Department of Education to discuss further. Our poster (or a link to it) was circulated to all schools nationally within Ireland, with accompanying text regarding the need for biohazard management awareness. The IMT has also separately contacted the Department of Education and the National Science competitions to explain the risks associated with this kind of science project and that clear prohibition of blood related research projects should apply for any national science competition.

\section{GP209 EVALUATING PARENT TRAINING PROGRAMMES FOR SELF-HARM - A FIVE YEAR INVESTIGATION OF THE EFFECTIVENESS OF THE SPACE (SUPPORTING PARENTS AND CARERS OF YOUNG PEOPLE WITH SELF-HARM THOUGHTS AND BEHAVIOURS) PROGRAMME}

${ }^{1}$ Carla Enge ${ }^{*},{ }^{1}$ Carole Boylan, ${ }^{1}$ Tessa McKenna, ${ }^{2}$ Sophia Morgan, ${ }^{1}$ Udo Reulbach,
${ }^{1,3}$ Elizabeth Barrett. ${ }^{1}$ Children's Unviersity Hospital, Temple Street, Dublin, Ireland; ${ }^{2}$ St
Patrick's Mental Health Services, Dublin, Ireland; ${ }^{3}$ University College Dublin, Dublin, Ireland

10.1136/archdischild-2019-epa.268

Background The family plays a pivotal role in the positive prognosis of young people who self-harm. However, parents and carers often feel ill-equipped to help their young person and are in need of support. The SPACE programme, a psycho-educational support group intervention, was developed by Children's University Hospital, Temple Street in Dublin, Ireland. It aims to empower parents to support their children through education, peer-support and skills development.

Aims and hypothesis Previous pilot studies have revealed positive findings for the SPACE (Supporting Parents And Carers) programme as a clinical intervention for self-harm. Consequently, the present study aimed to evaluate the effectiveness of the SPACE programme over a five year period in a realworld setting to determine its applicability and generalizability. Based on previous findings, it was hypothesised that participants' overall well-being would improve, evidenced by significant changes in levels of psychological distress, parenting satisfaction, perceived social support, and family communication. It was also hypothesised that the child's wellbeing would improve, evidenced by a lowering in total difficulties for the child as rated by the parent.

Methods A repeated-measures design with 260 participants was employed. Participants were parents and carers who attended the SPACE programme from 2013-2018. The effectiveness of SPACE was evaluated across five outcomes: psychological distress, parenting satisfaction, family communication, perceived social support, and parent-rated strengths and difficulties of the child. Questionnaires were administered at baseline, post-intervention and at three-month follow-up.

Results Data revealed significant reductions in participant psychological distress and parent-rated difficulties of the young person. Analysis also showed an increase in parental satisfaction. Perceived social support and family communication scores remained unchanged throughout.

Conclusions Data from both pilot and real-world studies suggest that the SPACE programme may be an effective clinical intervention to improve parent and child well-being, and may consequently contribute to improving prognosis for children who self-harm. Additionally, this evidence provides further support to the development of a clinical manual for the SPACE programme, with the aim of expanding its accessibility nationally and globally.

\section{GP210 BULLYNG AND CYBERBULLYNG IN SOUTHERN ITALY: A SCHOOL BASED STUDY}

${ }^{1}$ Anna Libera Latino*, ${ }^{2}$ Tiziana Carella, ${ }^{1}$ Rosa Pedale, ${ }^{1}$ Maria Teresa Vaccaro, ${ }^{1}$ Giuseppina Volpicelli. ${ }^{1}$ ASL-FG, Foggia, Italy; ${ }^{2}$ Free Psychologist, Foggia, Italy

\subsection{6/archdischild-2019-epa.269}

Background Bullying is the systematic abuse of power and is defined as aggressive behaviour or intentional harm-doing by peers carried out repeatedly with an imbalance of power. Bullying and cyberbullying are recognized risk factors for both short and long-term childhood and adolescent health: the aim of this cross-sectional study was to explore epidemiology of bullying and cybervictimization across seconddegree school in southern Italy, in order to plan detailed preventive strategies for their physical and psychosocial wellbeing.

Methods A cross-sectional school-based study was conducted in eight second-degree schools of Southern Italy, city of Foggia. Anonymous self-completed questionnaire was evaluated in 771 children, $51.7 \%$ female and $48.2 \%$ male, age ranging $12-$ 16 years. The questionnaire included evaluation of school well-being; child evaluation of bullying related aspects; forms of direct bullying, such as physical and verbal acts, or indirect bullying, as social exclusion; number of children who bullied or were being bullied; assisted bullying; cyberbullying. The project also included a dedicated training of teachers and family pediatricians to recognize alarm signals in this setting. Each school received their own results for awareness campaigns with parents and teachers.

Results As in previous Italian reports, about $31.62 \%$ were bullied during the previous 6 months; the higher the age, the lower the incidence of bullying (23.2\% versus $39.3 \%$ among lower age); direct bullying was the most common type $(52.45 \%)$ and the exclusion from the group was higher among girls (35\% versus $19.5 \%$ among males); victims rarely referred (only $44.75 \%$ ) to teachers their negative experiences. On the other hand, the considered geographic area showed specific features: physical bullying more frequent at higher age $(21 \%$ versus $13 \%$ among lower age); high percentage of group bullying: $44.6 \%$, mainly due to males;

$27.64 \%$ feel right a group revenge for an offense; low bully self-disclosure, $3.86 \%$ high percentage of cyberbullying, $24.4 \%$, with a low comprehension of cyberbullying as aggressive behavior and with more serious frequency item among lower age.

Conclusions Specific awareness and multidisciplinary approach is needed to limit bullying and cyberbullism negative consequences in children and adolescents: family pediatricians, teachers and parents need to cooperate in order to prevent health challenges for both victims and bullying and to ensure them a safe physical and psychosocial development. Pediatrician may be valid support for both adolescent psycho-social distress and families who live these problems. 